\title{
6D Physical Interaction with a Fully Actuated Aerial Robot
}

\author{
Markus Ryl1 ${ }^{1}$, Giuseppe Muscio $^{2}$, Francesco Pierri ${ }^{2}$, Elisabetta Cataldi ${ }^{3}$, Gianluca Antonelli ${ }^{3}$, Fabrizio Caccavale ${ }^{2}$ \\ and Antonio Franchi ${ }^{1}$
}

\begin{abstract}
This paper presents the design, control, and experimental validation of a novel fully-actuated aerial robot for physically interactive tasks, named Tilt-Hex. We show how the Tilt-Hex, a tilted-propeller hexarotor is able to control the full pose (position and orientation independently) using a geometric control, and to exert a full-wrench (force and torque independently) with a rigidly attached end-effector using an admittance control paradigm. An outer loop control governs the desired admittance behavior and an inner loop based on geometric control ensures pose tracking. The interaction forces are estimated by a momentum based observer. Control and observation are made possible by a precise control and measurement of the speed of each propeller. An extensive experimental campaign shows that the Tilt-Hex is able to outperform the classical underactuated multi-rotors in terms of stability, accuracy and dexterity and represent one of the best choice at date for tasks requiring aerial physical interaction.
\end{abstract}

\section{INTRODUCTION}

The current trends in robotics research require a stricter cooperation between human and robots, that often have to interact in a shared environment. Along this line, aerial robots have recently been exploited in tasks as, e.g., transportation [1], structure assembly and object grasping [2], and wall inspection [3], requiring not only autonomous flight but also the interaction with the environment. Physical interaction between human and/or the environment with multi-rotor aerial robots constitutes a particularly challenging scenario due to their intrinsic instability and typical underactuation.

To achieve physical interaction, aerial robots have been either equipped with complex robotic arms (see [4]-[6] and references therein) or tools rigidity attached to the aerial robot main structure as, e.g., in [2], [7]-[10]. The first approach exploits the redundancy brought by the manipulator to cope with the underactuation of the platform. This is obtained at the price of an increased cost, mechanical/control complexity, size, payload and a reduced endurance. The latter approach does not suffer from the aforementioned disadvantages, but it lacks of dexterity, because of the impossibility to control the pitch and roll independently from the lateral motion, and to exert lateral forces without necessarily tilt the whole structure. For these reasons the latter approach has

\footnotetext{
${ }^{1}$ LAAS-CNRS, Université de Toulouse, CNRS, Toulouse, France, markus.ryll@laas.fr, antonio.franchi@laas.fr

${ }^{2}$ University of Basilicata, School of Engineering, via dell' Ateneo Lucano, 10, Potenza, Italy, francesco.pierri@unibas.it, fabrizio.caccavale@unibas.it, giuseppe.muscio@unibas.it

${ }^{3}$ University of Cassino and Southern Lazio, Via Di Biasio 43, 03043 Cassino, Italy, e.cataldi@unicas.it, antonelli@unicas.it

This work has been partially funded by the European Union's Horizon 2020 research and innovation program under grant agreement No 644271 AEROARMS and the German Research Foundation (DFG).
}

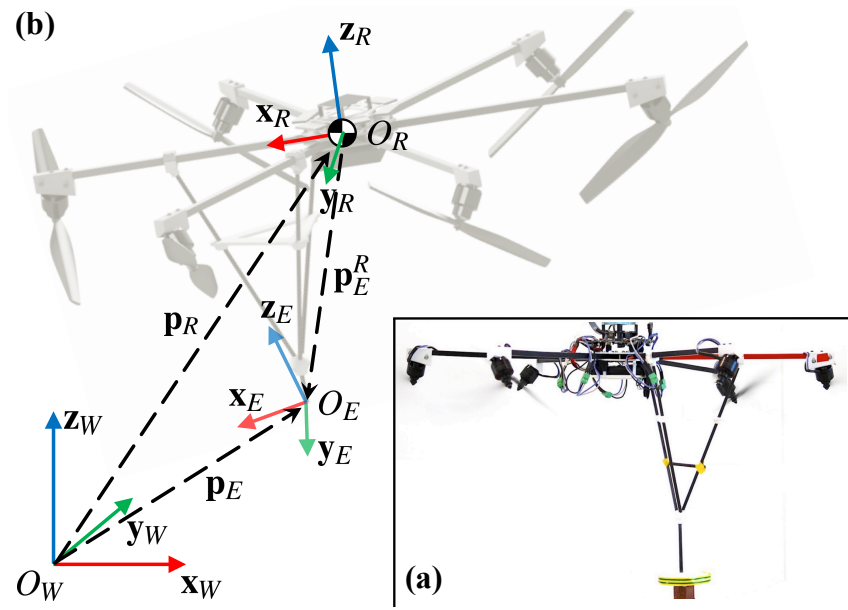

Fig. 1: a) The Tilt-Hex (NCFTP platform in-house developed at LAAS-CNRS) with the rigidly attached end-effector in contact with a force torque sensor used as ground truth. Notice how the interaction takes place far away and off-centered from the vehicle CoM. b) Schematic view of important frames and vectors.

been used so far only to perform simple interaction tasks (e.g, pick and place) with a gripper typically positioned close to the vehicle center of mass (CoM) which is not typically the best place to reach and/or to expose to physical interaction. In fact, it has been shown that in the presence of interactions with points of the structure other than the CoM, the internal dynamics of underactuated multi-rotors is not guaranteed to be stable, and is not, in general, easy to stabilize [9].

Here we propose a solution overcoming the lack of dexterity and unstable internal dynamics by using a multi-rotor robot with non-collinear fixedly-tilted propellers (NCFTP) instead of the more common collinear fixedly-tilted propeller (CFTP) architectures. In NCFTP platforms, which appeared in the robotics literature only recently (see, e.g., [11]-[14]), full-actuation is achieved by a proper design of the propeller positions and orientations. In this way they are able to control independently their orientational and translational accelerations when unconstrained, or any of the six components of the exerted wrench when in contact, thus allowing full and dexterous 6D force control, which makes them much more suited for physical interaction tasks than standard CFTP platforms. In order to control the interaction we use an admittance approach [15] that uses the wrench estimated by a wrench observer [16], [17]. The whole system is able to work at an unprecedented performance level thanks to the accurate control of the propeller speed provided by [18].

The paper is organized as follows. In Sec. II we first introduce a generic model for NCFTP aerial systems and 
afterwards we model the Tilt-Hex. In Sec. III we introduce the complete admittance control framework and we present the single components, namely the inner loop geometric pose controller, the wrench observer and the outer loop admittance filter. Then, in Sec. IV we present the hard-/software architecture. The conducted experiments are presented in Sec. V. Finally, Sec. VI concludes the paper with a summary of the results and an outline of future works.

\section{SySTEM MOdELING}

We consider as aerial robot a fully actuated aerial vehicle equipped with an arbitrarily mounted end-effector tool. The presented physical interaction framework is generic for any fully actuated system, able to track a full-pose trajectory with the end-effector. We shall start with the generic parts of the modeling (see Sec. II-.1) and we shall afterwards present the instantiation of this general model for the NCFTP hexarotor used in the experiments (see Sec. II-.2).

1) General Modeling: Let us denote with $\mathscr{F}_{W}$ the inertial world frame, whose axes (unit vectors) and origin are indicated with $\left\{\mathbf{x}_{W}, \mathbf{y}_{W}, \mathbf{z}_{W}\right\}$ and $O_{W}$, respectively (see Fig. 1). Then we denote with $\mathscr{F}_{R}: O_{R}-\left\{\mathbf{x}_{R}, \mathbf{y}_{R}, \mathbf{z}_{R}\right\}$ the body frame rigidly attached to the robot, where $O_{R}$ coincides with the robot's CoM (including the end-effector). The position of $O_{R}$ expressed in $\mathscr{F}_{W}$ is denoted by $\mathbf{p}_{R} \in \mathbb{R}^{3}$. Let us denote with $\mathscr{F}_{E}: O_{E}-\left\{\mathbf{x}_{E}, \mathbf{y}_{E}, \mathbf{z}_{E}\right\}$ the end-effector frame that is also rigidly attached to the robot. The position of $O_{E}$ in $\mathscr{F}_{W}$ and in $\mathscr{F}_{R}$ are denoted by $\mathbf{p}_{E} \in \mathbb{R}^{3}$ and $\mathbf{p}_{E}^{R} \in \mathbb{R}^{3}$, respectively, where $\mathbf{p}_{E}^{R}$ is constant over time.

We generally denote with $\mathbf{R}_{\circ}^{\star} \in S O(3)$ (where $S O(3)=$ $\left\{\mathbf{A} \in \mathbb{R}^{3 \times 3} \mid \mathbf{A A}^{\mathrm{T}}=\mathbf{I}\right\}$ ) the rotation matrix expressing the orientation of a frame $\mathscr{F}_{\circ}$ with respect to a frame $\mathscr{F}_{\star}$. If $\star$ is omitted it is intended as if $\star=W$. Generically, $\boldsymbol{\omega}_{\circ} \in \mathbb{R}^{3}$ denotes the angular velocity of $\mathscr{F}_{\circ}$ w.r.t. $\mathscr{F}_{W}$, expressed in $\mathscr{F}_{\circ}$. Given all the previous definitions, the orientation kinematics of the robot and the end effector are then expressed by

$$
\dot{\mathbf{R}}_{R}=\mathbf{R}_{R}\left[\boldsymbol{\omega}_{R}\right]_{\times} \quad \text { and } \quad \dot{\mathbf{R}}_{E}=\mathbf{R}_{E}\left[\boldsymbol{\omega}_{E}\right]_{\times},
$$

respectively, where $[\bullet]_{\times} \in S O(3)$ represents, in general, the skew symmetric matrix associated to any vector $\bullet \in \mathbb{R}^{3}$.

Using the Newton-Euler formalism, the equation of motion of the aerial robot can be expressed as

$$
\left[\begin{array}{c}
m \ddot{\mathbf{p}}_{R} \\
\mathbf{J} \dot{\boldsymbol{\omega}}_{R}
\end{array}\right]=\underbrace{-\left[\begin{array}{c}
m g \mathbf{e}_{3} \\
\boldsymbol{\omega}_{R} \times \mathbf{J} \boldsymbol{\omega}_{R}
\end{array}\right]}_{\mathbf{g}\left(\boldsymbol{\omega}_{R}\right)}+\left[\begin{array}{c}
\mathbf{f}^{W} \\
\boldsymbol{\tau}^{R}
\end{array}\right]+\mathbf{J}_{E}^{\mathrm{T}} \mathbf{w}_{E}^{e}
$$

with $m$ and $\mathbf{J} \in \mathbb{R}^{3 \times 3}$ representing the robot mass and its inertia matrix with respect to $O_{R}$ and expressed in $\mathscr{F}_{B}$, respectively, $g$ is the gravitational acceleration, and $\mathbf{f}^{W} \in$ $\mathbb{R}^{3}$ and $\tau^{R} \in \mathbb{R}^{3}$ are force and torque input. The external contact wrench arising at the end-effector expressed in $\mathscr{F}_{W}$ is denoted by $\mathbf{w}_{E}^{e} \in \mathbb{R}^{6}$ while its estimation (observation) is denoted by $\hat{\mathbf{w}}_{E}^{e}=\left[\begin{array}{llllll}\hat{f}_{E_{1}}^{e} & \hat{f}_{E_{2}}^{e} & \hat{f}_{E_{3}}^{e} & \hat{\tau}_{E_{1}}^{e} & \hat{\tau}_{E_{2}}^{e} & \hat{\tau}_{E_{3}}^{e}\end{array}\right]^{\mathrm{T}} \in \mathbb{R}^{6}$.

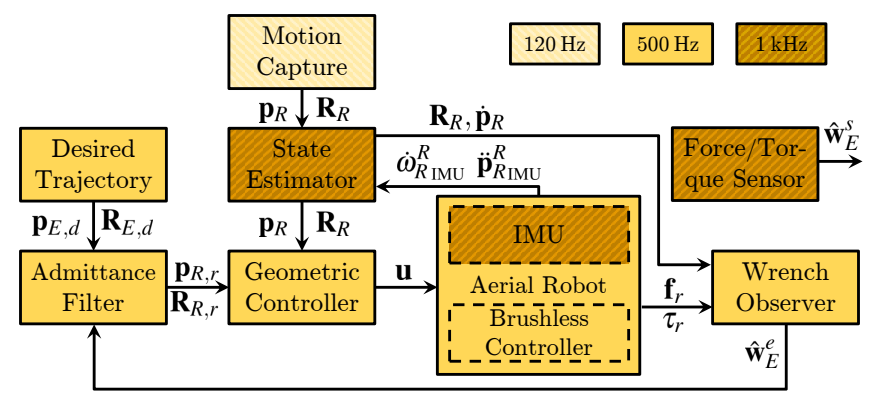

Fig. 2: Signal block diagram of the control framework. The runtime frequency is highlighted. For clarity higher derivatives of the signals have been omitted. The cascaded structure of the pose controller has been omitted as well.

2) Tilt-Hex: The NCFTP platform used in the experiments is a hexarotor structure where propellers are mounted on 6 equidistant/equally-spaced bars in the $\mathrm{x}-\mathrm{y}$-plane of $\mathscr{F}_{R}$. To achieve the full actuation each propeller has been fixated by a rigid adapter that tilts it (see Fig. 1).

The total force applied to $O_{R}$, expressed in $\mathscr{F}_{W}$ is

$$
\mathbf{f}^{W}(\mathbf{u})=\mathbf{R}_{R} \mathbf{F}_{1} \mathbf{u}
$$

where $\mathbf{u}=\left[u_{1} \ldots u_{6}\right]^{\mathrm{T}}$ is the vector of the 6 squared propeller spinning velocities and $\mathbf{F}_{1} \in \mathbb{R}^{3 \times 6}$ incorporates the physical properties of the hexarotor (i.e., propeller tilting angles and thrust coefficients). Similarly, the total moment is

$$
\tau^{R}(\mathbf{u})=\mathbf{F}_{2} \mathbf{u}
$$

where $\mathbf{F}_{2} \in \mathbb{R}^{3 \times 6}$ incorporates all the physical properties. A detailed derivation of the model and of $\mathbf{F}_{1}$ and $\mathbf{F}_{2}$ can be found in [11]. By replacing (3) and (4) in (2) we obtain

$$
\begin{gathered}
{\left[\begin{array}{c}
m \ddot{\mathbf{p}}_{R} \\
\mathbf{J} \dot{\boldsymbol{\omega}}_{R}
\end{array}\right]=\mathbf{g}\left(\boldsymbol{\omega}_{R}\right)+\left[\begin{array}{c}
\mathbf{R}_{R} \mathbf{F}_{\mathbf{1}} \\
\mathbf{F}_{\mathbf{2}}
\end{array}\right] \mathbf{u}=\mathbf{g}\left(\boldsymbol{\omega}_{R}\right)+\mathbf{F}\left(\mathbf{R}_{R}\right) \mathbf{u} .} \\
\text { III. CONTROLLER }
\end{gathered}
$$

In this section we describe the single components of the controller. The control framework is based on an outer loop admittance control and an inner loop full-pose geometric controller (see Fig. 2). The state of the aerial robot is estimated by a UKF that fuses the IMU acceleration and angular velocity measurements with the position and orientation from a pose sensor (in our case a motion capture system, which could be easily replaced with an onboard camera using a Perspective-n-Point (PnP) algorithm). The interaction torques and forces are estimated by a wrench observer.

\section{A. Pose Controller}

The goal of the inner loop control law is to compute the six desired propeller spinning rates $\mathbf{u}$ that let $\mathbf{p}_{R}$ and $\mathbf{R}_{R}$ track at best an arbitrary full-pose reference trajectory $\left(\mathbf{p}_{R, r}, \mathbf{R}_{R, r}\right): \mathbb{R} \rightarrow \mathbb{R}^{3} \times S O(3)$. Our controller is composed by an inner control loop (attitude controller) and outer control loop (position controller). The controller terminates with a force-torque mapper that computes the actual input $\mathbf{u}$ based on the reference control force $\mathbf{f}_{r} \in \mathbb{R}^{3}$ and reference control moment $\tau_{r} \in \mathbb{R}^{3}$ provided by the position and attitude controller, respectively. The controller has been thoroughly discussed in [19] we will therefore now present it briefly. 
1) Attitude controller: The attitude controller takes as input the reference orientation and the measured attitude state $\mathbf{R}_{R}, \boldsymbol{\omega}_{R}$ to compute the reference control torque $\tau_{r} \in \mathbb{R}^{3}$ as

$$
\boldsymbol{\tau}_{r}=\boldsymbol{\omega}_{R} \times \mathbf{J} \boldsymbol{\omega}_{R}-\mathbf{K}_{R} \mathbf{e}_{R}-\mathbf{K}_{\omega} \boldsymbol{\omega}_{R}
$$

where $\mathbf{K}_{R}, \mathbf{K}_{\omega}$ are positive definite gain matrixes, and the orientation error $\mathbf{e}_{R}$ is defined as

$$
\mathbf{e}_{R}=\frac{1}{2}\left({ }^{* \mathrm{~T}} \mathbf{R}_{R, r} \mathbf{R}_{R}-\mathbf{R}_{R}^{\mathrm{T}} \mathbf{R}_{R, r}^{*}\right)^{\vee}
$$

and $\bullet \vee$ is the vee map from $s o(3)$ to $\mathbb{R}^{3}$, see [19]. Note that $\mathbf{R}_{R, r}^{*}$ and $\mathbf{R}_{R, r}$ are not necessarily identical. The vector $\boldsymbol{\tau}_{r}$ is then passed to the force-torque mapper.

2) Position controller: The position controller takes as input the full-pose reference trajectory and the measured translational state $\mathbf{p}_{R}, \dot{\mathbf{p}}_{R}$ and produces as output the desired


and the reference control force $\mathbf{f}_{r} \in \mathbb{R}^{3}$ (sent directly to the force-torque mapper). Given the position tracking errors

$$
\mathbf{e}_{p}=\mathbf{p}_{R}-\mathbf{p}_{R, r}, \quad \text { and } \quad \mathbf{e}_{v}=\dot{\mathbf{e}}_{p}=\dot{\mathbf{p}}_{R}-\dot{\mathbf{p}}_{R, r}
$$

the reference force vector is computed as

$$
\mathbf{f}_{r}=m\left(\ddot{\mathbf{p}}_{r}+g \mathbf{e}_{3}-\mathbf{K}_{p} \mathbf{e}_{p}-\mathbf{K}_{v} \mathbf{e}_{v}\right),
$$

where $\mathbf{K}_{p}$ and $\mathbf{K}_{v}$ are positive definite gain matrixes.

The reason why we introduced a new reference orientation $\mathbf{R}_{R, r}^{*}$ in the latter section that is not necessarily equal to $\mathbf{R}_{R, r}$ is that the force $\mathbf{f}_{i}$ of every single propeller is naturally bounded by physical limits producing all together a polytope of feasible $3 \mathrm{D}$ forces that the aerial robot can exert. The polytope of the Tilt-Hex has its largest force along $\mathbf{z}_{R}$. In case that $\mathbf{f}_{r}$ in (9) is not implementable while also tracking the reference orientation, the controller seeks a novel $\stackrel{*}{\mathbf{R}}_{R, r}$ as close as possible to $\mathbf{R}_{R, r}$ such that $\mathbf{f}_{r}$ is contained in the feasible force polytope.

3) Force-torque mapper: The force-torque mapper takes as inputs $\mathbf{f}_{r}$ and $\tau_{r}$ and computes a feasible $\mathbf{u}$ as:

$$
\mathbf{u}=\left[\begin{array}{c}
\mathbf{R}_{R} \mathbf{F}_{1} \\
\mathbf{F}_{2}
\end{array}\right]^{-1}\left[\begin{array}{c}
\mathbf{f}_{r} \\
\boldsymbol{\tau}_{r}
\end{array}\right]
$$

To sum up the full controller in words, we aim at tracking a full-pose reference trajectory: if this is not feasible as the control output $\mathbf{u}$ would violate the minimum or maximum forces of any propeller $u_{i}$, we seek to find an $\mathbf{R}_{R, r}$ such that the input constraints are obeyed.

\section{B. Wrench Observer}

Let $\boldsymbol{v}_{R} \in \mathbb{R}^{6}$ be the twist (generalized velocity) of the aerial robot CoM, defined as $\boldsymbol{v}_{R}=\left[\begin{array}{ll}\dot{\mathbf{p}}_{R}^{\mathrm{T}} & \mathbf{R}_{R} \boldsymbol{\omega}_{R}^{\mathrm{T}}\end{array}\right]^{\mathrm{T}}$, it is possible to write (2) in Lagrangian form as

$$
\mathbf{M}\left(\mathbf{R}_{R}\right) \dot{\boldsymbol{v}}_{R}+\mathbf{C}\left(\mathbf{R}_{R}, \boldsymbol{\omega}_{R}\right) \boldsymbol{v}_{R}+\mathbf{g}_{R}=\overline{\mathbf{F}}\left(\mathbf{R}_{R}\right) \mathbf{u}-\mathbf{w}_{R}^{e},
$$

where, both linear and angular momenta are expressed in $\mathscr{F}_{W}, \mathbf{M}\left(\mathbf{R}_{R}\right)$ and $\mathbf{C}\left(\mathbf{R}_{R}, \boldsymbol{\omega}_{R}\right)$ are the inertia and Coriolis/centrifugal term matrices

$\mathbf{M}\left(\mathbf{R}_{R}\right)=\left[\begin{array}{cc}m \mathbf{I}_{3} & O_{3} \\ O_{3} & \overline{\mathbf{J}}\end{array}\right], \mathbf{C}\left(\mathbf{R}_{R}, \boldsymbol{\omega}_{R}\right)=\left[\begin{array}{cc}O_{3} & O_{3} \\ O_{3} & {\left[\mathbf{R}_{\mathbf{R}} \boldsymbol{\omega}_{R}\right]_{\times} \overline{\mathbf{J}}}\end{array}\right]$, with $\overline{\mathbf{J}}=\mathbf{R}_{R} \mathbf{J} \mathbf{R}_{R}^{\mathrm{T}}$ being the inertial tensor w.r.t. $O_{R}$ expressed in $\mathscr{F}_{W} ; \mathbf{g}_{R}=\left[m g \mathbf{e}_{3}^{\mathrm{T}} \mathbf{0}_{3}^{\mathrm{T}}\right]^{\mathrm{T}}$ is the vector collecting the gravity terms, $\overline{\mathbf{F}}=\left[\begin{array}{ll}\mathbf{F}_{1}^{\mathrm{T}} \mathbf{R}_{R}^{\mathrm{T}} & \mathbf{F}_{2}^{\mathrm{T}} \mathbf{R}_{R}^{\mathrm{T}}\end{array}\right]^{\mathrm{T}}$, and

$$
\mathbf{w}_{R}^{e}=\mathbf{H}_{E}\left(\mathbf{R}_{R}\right) \mathbf{w}_{E}^{e}, \quad \mathbf{H}_{E}\left(\mathbf{R}_{R}\right)=\left[\begin{array}{cc}
\mathbf{I}_{3} & {\left[\mathbf{R}_{R} \mathbf{p}_{E}^{R}\right]_{\times}} \\
\mathbf{O}_{3} & \mathbf{I}_{3}
\end{array}\right] .
$$

Equation (12) suggests that the term $\mathbf{w}_{R}^{e}$ represents the effect on the robot CoG of the wrench $\mathbf{w}_{E}^{e}$ acting on the tool tip. In order to estimate the interaction wrench, exerted by the tool-tip on the external environment, a momentum-based observer [16] has been designed. The generalized momentum $\mathbf{q} \in \mathbb{R}^{6}$ can be computed as

$$
\mathbf{q}=\mathbf{M}\left(\mathbf{R}_{R}\right) \dot{\boldsymbol{v}}_{R}
$$

By exploiting the property of the inertia matrix

$$
\dot{\mathbf{M}}\left(\mathbf{R}_{R}\right)=\mathbf{C}\left(\mathbf{R}_{R}, \boldsymbol{\omega}_{R}\right)+\mathbf{C}^{\mathrm{T}}\left(\mathbf{R}_{R}, \boldsymbol{\omega}_{R}\right),
$$

the time-derivative of (13) can be expressed as

$$
\dot{\mathbf{q}}=\mathbf{C}^{\mathrm{T}}\left(\mathbf{R}_{R}, \boldsymbol{\omega}_{R}\right) \boldsymbol{v}_{R}-\mathbf{g}_{R}+\overline{\mathbf{F}} \mathbf{u}+\mathbf{w}_{R}^{e} .
$$

Let $\hat{\mathbf{w}}_{R}^{e}, \in \mathbb{R}^{6}$ be the residual vector, defined as follows

$\hat{\mathbf{w}}_{R}^{e}=\mathbf{K}_{I}\left[\left(\mathbf{q}(t)-\mathbf{q}\left(t_{0}\right)\right)-\int_{t_{0}}^{t}\left(\mathbf{C}^{\mathrm{T}}\left(\mathbf{R}_{R}, \boldsymbol{\omega}_{R}\right) \boldsymbol{v}_{R}+\overline{\mathbf{F}} \mathbf{u}-\mathbf{g}_{R}+\hat{\mathbf{w}}_{R}^{e}\right) \mathrm{d} \tau\right]$,

where $t$ and $t_{0}$ are the current and initial time instant respectively, $\mathbf{K}_{I}$ is a positive definite gain matrix. By reasonably assuming that $\boldsymbol{v}_{R}\left(t_{0}\right)=\mathbf{0}_{6}$, it implies that $\mathbf{q}\left(t_{0}\right)$ is null as well. By taking the time derivative of (16), through (15), the following dynamics for the residual vector is obtained

$$
\dot{\hat{\mathbf{w}}}_{R}^{e}+\mathbf{K}_{I} \hat{\mathbf{w}}_{R}^{e}=\mathbf{K}_{I} \mathbf{w}_{R}^{e}
$$

where $\mathbf{w}_{R}^{e}$ is computed from (11). Equation (17) is a first order low-pass dynamic system: it can be easily recognized that $\hat{\mathbf{w}}_{R}^{e} \rightarrow \mathbf{w}_{R}^{e}$ when $t \rightarrow \infty$ and with $\mathbf{K}_{I} \simeq \infty$ it yields $\hat{\mathbf{w}}_{R}^{e} \simeq$ $\mathbf{w}_{R}^{e}$. Thus, a proper choice of $\mathbf{K}_{I}$ lets the observer achieve a good estimation of $\mathbf{w}_{R}^{e}$ while, at the same time, it filters out the high-frequency noise. Once $\hat{\mathbf{w}}_{R}^{e}$ is known, the estimated wrench acting on the tool tip, $\hat{\mathbf{w}}_{E}^{e}$ is computed as

$$
\hat{\mathbf{w}}_{E}^{e}=\mathbf{H}_{E}^{-1}\left(\mathbf{R}_{R}\right) \hat{\mathbf{w}}_{R}^{e} \text {. }
$$

\section{Admittance Filter}

In order to achieve bounded internal forces, a compliant behavior of the end-effector with respect to interaction generalized forces should be adopted. The desired trajectory of the end-effector, $\left(\mathbf{p}_{E, d}, \mathbf{R}_{E, d}, \boldsymbol{v}_{E, d}, \dot{\boldsymbol{v}}_{E, d}\right)$, given by an offline planner, represents the input of the admittance filter, that computes a new reference trajectory $\left(\mathbf{p}_{E, r}, \mathbf{R}_{E, r}, \boldsymbol{v}_{E, r}, \dot{\boldsymbol{v}}_{E, r}\right)$ for the end-effectors via the following dynamic system

$$
\mathbf{M}_{E} \Delta \dot{\boldsymbol{v}}_{E}+\mathbf{D}_{E} \Delta \boldsymbol{v}_{E}+\mathbf{K}_{E} \mathbf{e}_{E}=\hat{\mathbf{w}}_{E}^{e}
$$

which is the equation of a 6-DoF mechanical impedance [20] of inertia $\mathbf{M}_{E}$, damping $\mathbf{D}_{E}$ and stiffness $\mathbf{K}_{E}$ : those matrices are all positive-definite and suitably chosen in a way to impose an over-damped behavior to the system. The other terms in (19) are the velocity error $\Delta \boldsymbol{v}_{E}=\boldsymbol{v}_{E, d}-\boldsymbol{v}_{E, r}$ and the pose error $\mathbf{e}_{E}=\left[\mathbf{p}_{E, d}-\mathbf{p}_{E, r}, \Delta \boldsymbol{\xi}_{E}\right]$, being $\Delta \boldsymbol{\xi}_{E}$ the vector 
part of the unit quaternion extracted from the rotation matrix $\mathbf{R}_{E, d} \mathbf{R}_{E, r}^{\mathrm{T}}$ representing the orientation error.

Once the reference trajectory of the end-effector has been computed it should be expressed in terms of CoM reference trajectory in order to be tracked by the inner loop pose controller. The reference position and orientation of the robot are then computed (see Fig. 1) as

$$
\mathbf{p}_{R, r}=\mathbf{p}_{E, r}-\mathbf{R}_{R, r} \mathbf{p}_{E}^{R}, \quad \mathbf{R}_{R, r}=\mathbf{R}_{E, r} \mathbf{R}_{R}^{E},
$$

while the CoM reference velocities and accelerations are obtained by taking the time derivatives of (20).

\section{EXPERIMENTAL SETUP}

The Tilt-Hex robot is a LAAS-CNRS development based on off-the-shelf aluminum components, carbon fiber bars for the end-effector and 3D-printed structural components. The robot diameter (rotor hub to rotor hub) is $0.8 \mathrm{~m}$. The total mass is $m=1.8 \mathrm{~kg}$ including the end-effector and the distance from the center of mass to the tool tip is $\mathbf{p}_{E}^{R}=\left[\begin{array}{llll}0.12 & 0 & 0.4\end{array}\right]^{\mathrm{T}} \mathrm{m}$. The principal components of the inertia tensor, obtained by a detailed CAD model, have been identified as $\mathbf{J}=$ $\operatorname{diag}(11.5,11.4,19.4) 10^{-6} \mathrm{~kg} \mathrm{~m}^{2}$. The propeller tilting angles are $\alpha=30^{\circ}$ and $\beta=10^{\circ}$ (see [11] for the angle definition). which guarantee a well balanced choice between maximum lateral forces and losses due to internal forces.

The six 12 inch propellers are driven by six MK3638 brushless motors by MikroKopter. The maximum lift force per propeller is $12 \mathrm{~N}$. The hardware of the ESC (electronic speed control) unit driving each motors is the BL-Ctrl-2.0 from MikroKopter. The software is an in-house developed firmware that performs closed-loop spinning frequency control and accepts a desired spinning frequency at $1 \mathrm{kHz}$ [18]. At the same rate it provides a measurement of the current spinning frequency, that it is used in the wrench observer.

The on-board inertial measurement unit provides accelerometer and gyroscope measurements. An external motion capture system (Optitrack MoCap) based on optical markers has been used to retrieve the platform's position and orientation (notice that a $\mathrm{PnP}$ algorithm and an onboard camera could easily replace the MoCap). On the ground a 3D force torque sensor (ATI Mini45) has been used to validate off-line the estimations of the wrench observer. The MoCap measurements $(120 \mathrm{~Hz})$ are fused via a UKF state estimator with the IMU measurements $(1 \mathrm{kHz})$ thus obtaining a full state estimate at $1 \mathrm{kHz}$.

\section{EXPERIMENTAL RESUlTS}

The experimental setup described in Section IV has been adopted for testing the performance of the proposed scheme in the presence of interaction of the aerial robot with the environment - both objects and human subjects. We encourage the reader to watch the multimedia attachment to appreciate the experiments in action. For the reader's convenience the orientations corresponding to a rotation matrix are displayed in the plots using the common roll, pitch and yaw angles with the following convention: $\mathbf{R}_{\bullet} \rightarrow \phi_{\bullet}, \theta_{\bullet}, \psi_{\bullet}$. Furthermore the single components of position or error vectors are marked with indices using the following convention: $\left[[]_{x}[]_{y}[]_{z}\right]^{T}$.

\begin{tabular}{|l|c|c|c|c|}
\hline & & Exp. 1 & Exp. 2a & Exp. 2b \\
\hline \multirow{4}{*}{$\begin{array}{l}\text { Pose } \\
\text { Controller }\end{array}$} & $\mathbf{K}_{p}$ & 12 & 14 & 14 \\
\cline { 2 - 5 } & $\mathbf{K}_{v}$ & 4 & 8 & 8 \\
\cline { 2 - 5 } & $\mathbf{K}_{R}$ & 10 & 8 & 8 \\
\cline { 2 - 5 } & $\mathbf{K}_{\omega}$ & 1.4 & 1.4 & 1.4 \\
\hline \multirow{4}{*}{$\begin{array}{l}\text { Filmittance } \\
\end{array}$} & $\mathbf{M}_{E_{P}}$ & 1 & 1 & 4 \\
\cline { 2 - 5 } & $\mathbf{D}_{E_{P}}$ & 50 & 2 & 55 \\
\cline { 2 - 5 } & $\mathbf{K}_{E_{R}}$ & 80 & 4 & 80 \\
\cline { 2 - 5 } & $\mathbf{M}_{E_{R}}$ & 1 & 1 & 1 \\
\cline { 2 - 5 } & $\mathbf{D}_{E_{R}}$ & 50 & 12 & 7 \\
\cline { 2 - 5 } & $\mathbf{K}_{E_{R}}$ & 100 & 35 & 15 \\
\hline
\end{tabular}

TABLE I: Parameters used in the four experiments. Each value represents the multiplier of a $3 \times 3$ identity matrix.

\section{A. Exp. 1: Off-Centered Pushing and Wrench Estimator Test}

The first case study consists of an interaction of the endeffector tool with a planar surface (see Fig. 1). In order to achieve the interaction, a trajectory has been chosen such that the end-effector pushes vertically the surface, connected to a force/torque sensor, whose measurements represent the ground-truth. The goal is twofold: i) to show, by direct comparison with the ground truth, that the estimated force is a reliable estimate of the real one; $i$ i) to prove that, thanks to the full actuation, the tool can interact in a safe, stable, and non-oscillatory way through a point that is offset and far away from the CoM. Such behavior is impossible to achieve for a standard underactuated platform. The parameter values for the admittance filter are reported in Tab. I.

The planned desired trajectory of the end-effector and the reference one are reported in Fig. 3-1 (where '-j' stands for ' $\mathrm{j}$-th row', in this case ' $\mathrm{j}=1$ '). Between $27 \mathrm{~s}$ and $61 \mathrm{~s}$, the desired trajectory pierces the surface and the force/torque sensor measures an interaction force along the $\mathbf{z}_{W}$ axis. Fig. 3-5 shows the component of $\hat{\mathbf{w}}_{E}^{s}$ along $\mathbf{z}_{W}$ measured by the sensor (blue line) and the corresponding component of $\hat{\mathbf{w}}_{E}^{e}$ estimated via the wrench observer (16) (red line): the estimation is very close to the ground truth, with a maximum error of about $0.3 \mathrm{~N}$ (i.e., less than the $10 \%$ of the force value). The discrepancy between the planned desired trajectory and the reference one given by the admittance filter along $\mathbf{z}_{W}$ grows at the beginning of the contact phase, up to a value of about $0.05 \mathrm{~m}$ (see Fig. 3-2), and moves to zero when the contact force vanishes. Along the other axes it is very close to zero. In Fig. 3-3 and Fig. 3-4 inner pose control position and orientation errors are reported (see Section IIIA) namely the errors between the reference trajectory given by the admittance filter and the actual one: after the initial phase, both the position and orientation errors are very close to zero, also in the presence of contact. Thus, the controller is correctly behaving and the admittance filter is correctly tuned, since it limits the interaction forces without destabilizing the motion controller.

\section{B. Exp. 2: Disturbance Injection through Rope Pulling}

In this second experiment, the performance of the proposed scheme has been evaluated in the presence of an external disturbance injected by a human by pulling and releasing a rope attached to the aerial platform, and with different tuning of the admittance parameters. The rope has been fixated on the end-effector structure where it opens 


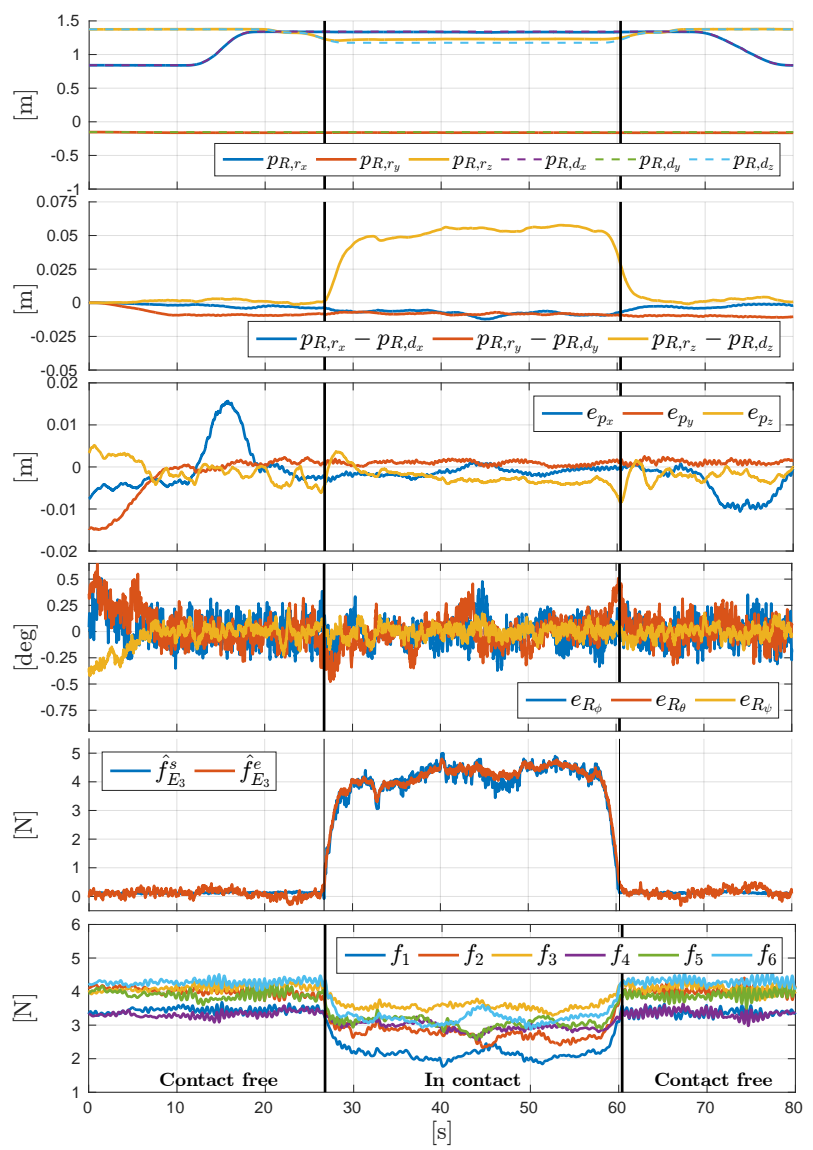

Fig. 3: Interaction through an off-centered point with flat surface attached to a force sensor.

into the triangular structure (at the level of the lowest visual marker in Fig 1) being $10 \mathrm{~cm}$ above the end-effector tip but $30 \mathrm{~cm}$ below the center of mass (and off centered w.r.t. to it). With respect to the end-effector tip this position allows to generate a combined force and torque.

Firstly the stiffness matrix $\mathbf{K}_{E}$ in (19) was tuned to make the system highly compliant with respect to interaction forces and relatively stiff respect to interaction torques, by choosing small position and high orientation gains (for detailed values see Tab. I-Exp.2a). Thus, in the presence of the external disturbance, the platform has to keep the assigned orientation while large deviation from the desired position are requested. The desired position and orientation through these experiments was constant and horizontal at the origin. Figure 4 shows the obtained results: as expected, from Fig. 4-1 there is a large deviation of position under presence of the estimated forces $\hat{f}_{E_{2}}^{e}$ with peaks of $2 \mathrm{~N}$ (see Fig. 4-5), while the orientation remains almost horizontal despite interaction torques with peak values of about $2 \mathrm{Nm}$ about the $\mathbf{x}_{W}$ and $\mathbf{y}_{W}$ axes (see Fig. 4-4). Concerning the inner loop pose control errors, in this case larger errors are experienced due to the more challenging scenario and the dynamic change of the applied wrench, however the errors, after the initial phase, are still acceptable for a flying platform, reaching peak values of about $0.11 \mathrm{~m}$ for the position and $3^{\circ}$ for the orientation when the human pulls the rope. Again, thanks to the full actuation everything remains perfectly stable.

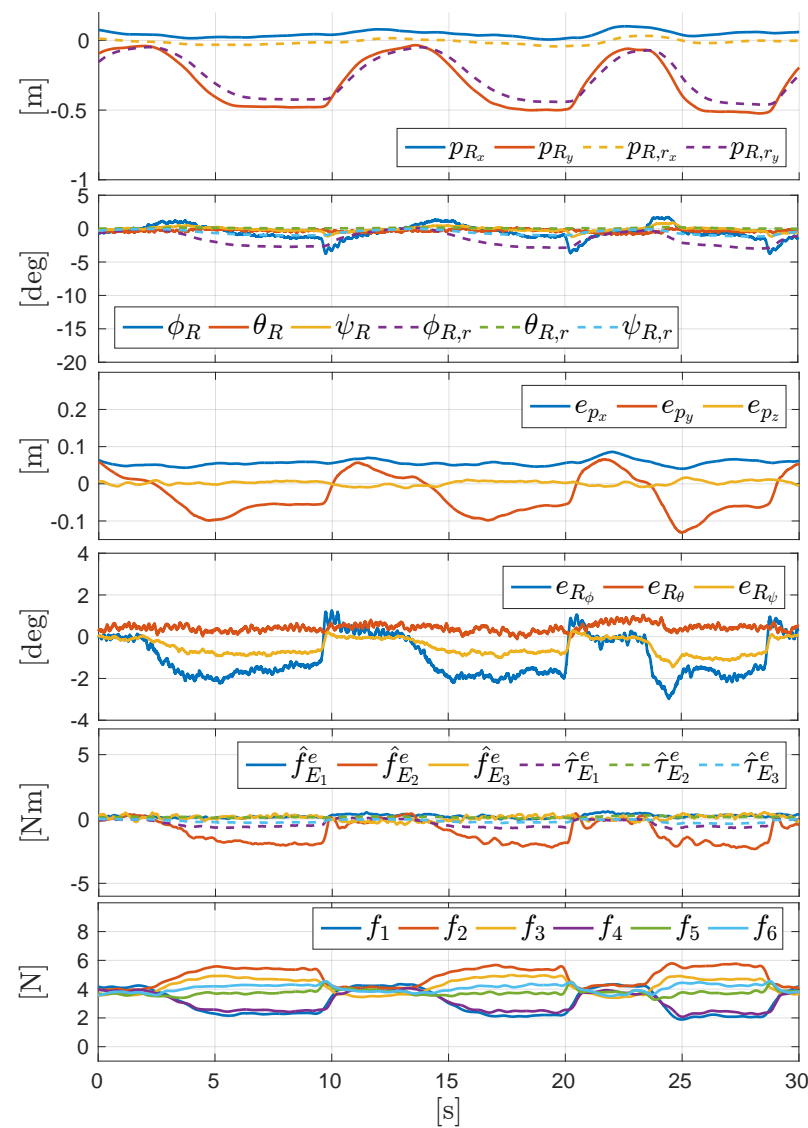

Fig. 4: External disturbance injected by human with a rope: compliant position/stiff orientation case.

Then, a different tuning of the stiffness matrix $\mathbf{K}_{E}$ was tested by choosing large position gains and small orientation gains (for detailed values see Tab. I-Exp.2b). In this way the system is made compliant with respect to interaction torques and stiff with respect to interaction forces. In this case, in the presence of the external disturbance, the platform has to keep the assigned position while a large deviation from the desired orientation is allowed. Figure 5 shows the experimental results: as expected, Fig. 5-2 shows a large deviation of the orientation, while the deviation of the position is reduced with respect to the previous scenario (although the exerted forces are double then before - see Fig. 5-5). Again the motion control error in Fig. 5-3 and 4 remain small.

Notice that in this second case the behavior of the robot is (on purpose) counterintuitive. While pulling the observers intuition would tell that the aerial robot will rotate about the CoM, however the admittance method, applied with respect to the end-effector tip, forces the aerial robot to rotate about the tip. This results in a rotation opposing the intuition. The interested reader is referred to the video. It is worth to notice that the platform remained stable thanks to the inner loop geometric control although we reached the saturation of rotor 1 (see Fig. 5-6). This experiment proves as well that the fully actuated platform, with the motion control described in Section III, is able to counteract large disturbances both in term of forces and torques without loss of stability. 


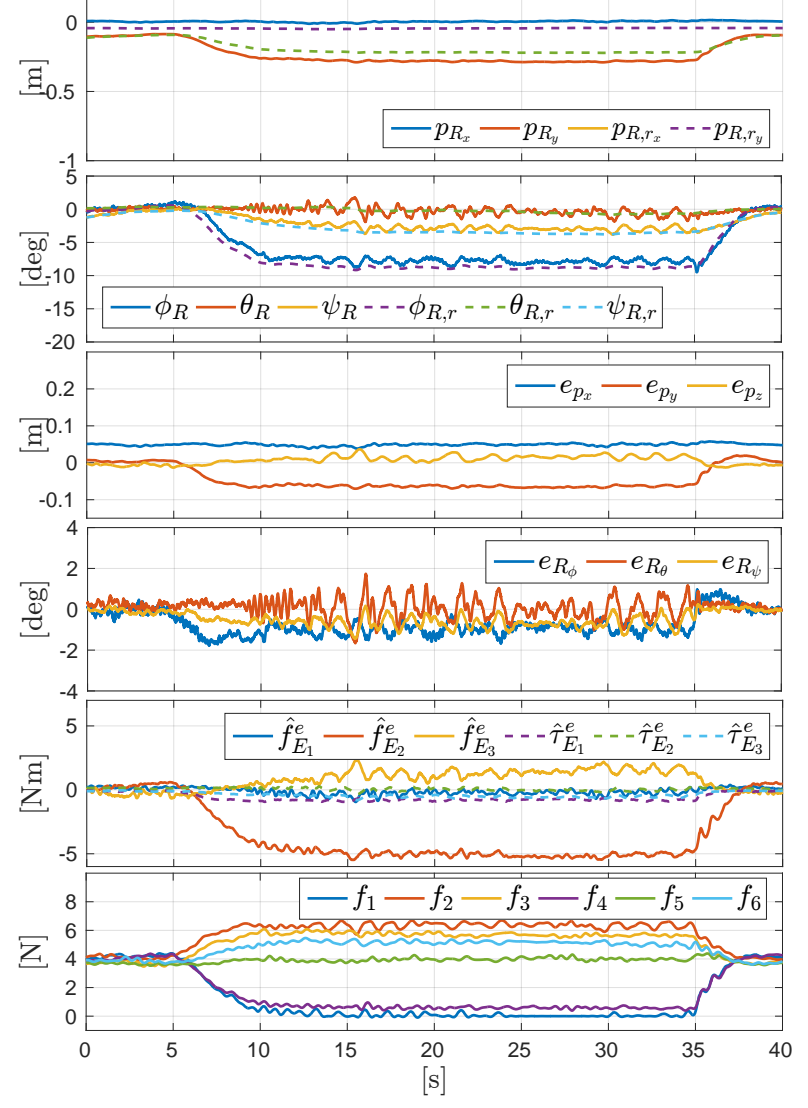

Fig. 5: External disturbance injected by a human with a rope: compliant orientation/stiff position case.

\section{SUMMARY}

In this paper the problem of physical interaction of a novel fully actuated aerial robot with the external environment has been tackled out with the aim of keeping interaction forces bounded and guaranteeing a stable behavior of the robotic system. The proposed aerial platform, developed at LAASCNRS and named Tilt-Hex, allows to control independently both linear and angular accelerations and, thus, to counteract any wrench during the contact with the environment. A two-loop control scheme has been designed: at the outer loop an admittance control scheme steers the system to a desired impedance behavior in the presence of interaction wrenches by computing suitable reference trajectories that are fed to a geometrically-based motion controller (the inner loop). Finally a relevant experimental campaign has been successfully conducted.

In the future we plan to make the robot more autonomous by using onboard sensors and also interfaceable with a human operator through a haptic (bilateral) shared control, as done, e.g., in [21] for a contact-free underactuated vehicle.

\section{REFERENCES}

[1] J. Fink, N. Michael, S. Kim, and V. Kumar, "Planning and control for cooperative manipulation and transportation with aerial robots," The International Journal of Robotics Research, vol. 30, no. 3, pp. 324-334, 2011.

[2] F. Augugliaro, S. Lupashin, M. Hamer, C. Male, M. Hehn, M. W. Mueller, J. S. Willmann, F. Gramazio, M. Kohler, and R. D'Andrea,
"The flight assembled architecture installation: Cooperative construction with flying machines," IEEE Control Systems Magazine, vol. 34, no. 4, pp. 46-64, 2014.

[3] M. Fumagalli, R. Naldi, A. Macchelli, R. Carloni, S. Stramigioli, and L. Marconi, "Modeling and control of a flying robot for contact inspection," in 2012 IEEE/RSJ Int. Conf. on Intelligent Robots and Systems, Vilamoura, Portugal, Oct 2012, pp. 3532-3537.

[4] G. Muscio, F. Pierri, M. A. Trujillo, E. Cataldi, G. Giglio, G. Antonelli, F. Caccavale, A. Viguria, S. Chiaverini, and A. Ollero, "Experiments on coordinated motion of aerial robotic manipulators," in 2016 IEEE Int. Conf. on Robotics and Automation, Stockholm, Sweden, May 2016, pp. 1224-1229.

[5] K. Baizid, G. Giglio, F. Pierri, M. Trujillo, G. Antonelli, F. Caccavale, A. Viguria, S. Chiaverini, and A. Ollero, "Behavioral control of unmanned aerial vehicle manipulator systems," Autonomous Robots, vol. 35 , no. 8, pp. 1-18, 2016.

[6] M. Tognon, B. Yüksel, G. Buondonno, and A. Franchi, "Dynamic decentralized control for protocentric aerial manipulators," in 2017 IEEE Int. Conf. on Robotics and Automation, Singapore, May 2017.

[7] G. Gioioso, M. Ryll, D. Prattichizzo, H. H. Bülthoff, and A. Franchi, "Turning a near-hovering controlled quadrotor into a 3D force effector," in 2014 IEEE Int. Conf. on Robotics and Automation, Hong Kong, China, May. 2014, pp. 6278-6284.

[8] B. Yüksel, C. Secchi, H. H. Bülthoff, and A. Franchi, "Reshaping the physical properties of a quadrotor through IDA-PBC and its application to aerial physical interaction," in 2014 IEEE Int. Conf. on Robotics and Automation, Hong Kong, China, May. 2014, pp. 62586265.

[9] H. Nguyen and D. Lee, "Hybrid force/motion control and internal dynamics of quadrotors for tool operation," in 2013 IEEE/RSJ Int. Conf. on Intelligent Robots and Systems, Tokyo, Japan, Nov. 2013, pp. 3458-3464.

[10] G. Gioioso, A. Franchi, G. Salvietti, S. Scheggi, and D. Prattichizzo, "The Flying Hand: a formation of uavs for cooperative aerial telemanipulation," in 2014 IEEE Int. Conf. on Robotics and Automation, Hong Kong, China, May. 2014, pp. 4335-4341.

[11] S. Rajappa, M. Ryll, H. H. Bülthoff, and A. Franchi, "Modeling, control and design optimization for a fully-actuated hexarotor aerial vehicle with tilted propellers," in 2015 IEEE Int. Conf. on Robotics and Automation, Seattle, WA, May 2015, pp. 4006-4013.

[12] R. Voyles and G. Jiang, "A nonparallel hexrotor UAV with faster response to disturbances for precision position keeping," in 2014 IEEE Int. Symp. on Safety, Security and Rescue Robotics, West Lafayette, IN, Oct. 2014, pp. 1-5.

[13] D. Brescianini and R. D'Andea, "Design, modeling and control of an omni-directional aerial vehicle," in 2016 IEEE Int. Conf. on Robotics and Automation, Stockholm, Sweden, May 2016, pp. 3261-3266.

[14] S. Park, J. J. Her, J. Kim, and D. Lee, "Design, modeling and control of omni-directional aerial robot," in 2016 IEEE/RSJ Int. Conf. on Intelligent Robots and Systems, Daejeon, South Korea, 2016, pp. 1570-1575.

[15] G. Antonelli, E. Cataldi, G. Muscio, M. Trujillo, Y. Rodriguez, F. Pierri, F. Caccavale, A. Viguria, S. Chiaverini, and A. Ollero, "Impedance control of an aerial-manipulator: Preliminary results," in 2016 IEEE/RSJ Int. Conf. on Intelligent Robots and Systems, Daejeon, South Korea, 2016, pp. 3848-3853.

[16] A. De Luca and R. Mattone, "Sensorless robot collision detection and hybrid force/motion control," in 2005 IEEE Int. Conf. on Robotics and Automation, Barcelona, Spain, Apr. 2005, pp. 999-1004.

[17] B. Yüksel, C. Secchi, H. H. Bülthoff, and A. Franchi, "A nonlinear force observer for quadrotors and application to physical interactive tasks," in 2014 IEEE/ASME Int. Conf. on Advanced Intelligent Mechatronics, Besançon, France, Jul. 2014, pp. 433-440.

[18] A. Franchi and A. Mallet, "Adaptive closed-loop speed control of BLDC motors with applications to multi-rotor aerial vehicles," in 2017 IEEE Int. Conf. on Robotics and Automation, Singapore, May 2017.

[19] M. Ryll, D. Bicego, and A. Franchi, "Modeling and control of FASTHex: a fully-actuated by synchronized-tilting hexarotor," in 2016 IEEE/RSJ Int. Conf. on Intelligent Robots and Systems, Daejeon, South Korea, Oct. 2016, pp. 1689-1694.

[20] B. Siciliano, L. Sciavicco, L. Villani, and G. Oriolo, Robotics: Modelling, Planning and Control. Springer, 2009.

[21] P. Stegagno, M. Basile, H. H. Bülthoff, and A. Franchi, "A semiautonomous UAV platform for indoor remote operation with visual and haptic feedback," in 2014 IEEE Int. Conf. on Robotics and Automation, Hong Kong, China, May. 2014, pp. 3862-3869. 